\title{
DOUBLE IDEALS IN COMPACT SEMIRINGS
}

\author{
MARTHA BERTMAN AND JOHN SELDEN
}

(Received 5 January 1972)

Communicated by G. B. Preston

By a topological semiring we mean a Hausdorff space $S$ together with two continuous associative operations on $S$ such that one (called multiplication) distributes across the other (called addition). That is, we insist that

$$
x(y+z)=x y+x z \text { and }(x+y) z=x z+y z
$$

for all $x, y$ and $z$ in $S$. Note that, in contrast to the purely algebraic situation $[1,2]$, we do not postulate the existence of an additive identity which is a multiplicative zero.

In this note we point out conditions under which the existence of such an element is equivalent to the double simplicity of the semiring. We also discuss maximal and minimal double ideals together with several examples.

We let $E[+]$ represent the set of additive idempotents. Similarly $E[\cdot]=$ $\left\{e: e^{2}=e\right\}$. An additive (multiplicative), ideal of a semiring is a non-empty set $I$ such that

$$
S+I \cup I+S \subseteq I(S I \cup I S \subseteq I) .
$$

The minimal additive or multiplicative ideal is denoted by $K[+]$ or $K[\cdot]$. For references on the properties of these sets, the reader may see $[4,5]$. We say a set $I$ is a double ideal if it is both an additive and a multiplicative ideal. A semiring containing no proper double ideal will be called doubly simple.

THEOREM 1. Let $S$ be a compact additively and multiplicatively commutative semiring. Then $S$ is doubly simple if and only if there is an element 0 in $S$ such that $0+x=x$ and $0 x=0$ for all $x \in S$.

Proof. Suppose $S$ is doubly simple. Notice that $E[+]$ is a multiplicative ideal of $S$ and thus contains the minimal such, $K[\cdot]$. Now according to $[4 ; \mathrm{pp}$. 97 and 98$], K[\cdot]$ is the union of multiplicative groups each of which is of the form $e S e$ for some $e$ in $E[\cdot] \cap K[\cdot]$. Consider one of these groups, eSe. Clearly, it is a compact subsemiring and thus contains a minimal additive ideal. This minimal additive ideal of $e S e$ must be a group since addition is commutative. On the other 
hand, it is additively idempotent and thus a single point, say $k$. Now $k+x=k$ for all $x \in e S e$. Hence

$$
k^{2}=k(k+e)=k^{2}+k e=k^{2}+k=k
$$

and we see that $k$ is a multiplicative idempotent. Therefore $e=k$. Now, if $y \in e S e$ and $y^{-1}$ is its multiplicative inverse in $e S e$, then we have

$$
e=e+y=y y^{-1}+y e=y\left(y^{-1}+e\right)=y e=y .
$$

Thus $e S e$ consists of the single point $e$, and $K[\cdot]$ is multiplicatively idempotent. But since $S$ is multiplicatively commutative, $K[\cdot]$ is a group. Thus $K[\cdot]$ is a single point 0 , such that $0 x=0$ for all $x \in \mathrm{S}$, and $0+0=0$. Now $0+S$ is a double ideal of $S$, so $0+S=S$.Thus if $x \in S$, there exists a $y$ in $S$ such that $x=S$ such that $x=0+y$. Therefore,

$$
0+x=0+(0+y)=0+y=x .
$$

On the other hand, suppose there is such an element 0 is $S$ and suppose $A$ is a double ideal. Clearly $0 \in A$ since 0 is the minimal multiplicative ideal. Now

$$
S=0+S \subseteq A+S \subseteq A
$$

and the proof is complete.

Example A. Here are several doubly simple semirings:

i) any ring;

ii) the real interval $[0,1]$ with $x \cdot y=x \wedge y$ and $x+y=x \vee y$;

iii) $[0,1]$ with ordinary multiplication and $x+y=x \vee y$;

iv) $[0,1]$ with $x+y=x \vee y$ and $x y=x$ for all $x, y$.

This last mentioned semiring illustrates the necessity of commutativity in Theorem 1, since it is doubly simple but lacks a multiplicative zero.

Note that the cartesian product of semirings is a semiring under coordinatewise addition and multiplication; and that the product of doubly simple semirings is again doubly simple. To see this, let $\left\{S_{a}\right\}$ be a collection of doubly simple semirings. Suppose tht $J$ is a double ideal of $\Pi_{\alpha} S_{\alpha}=S$ and $f$ is in $J$. Then $S+S f S+S \subseteq J$. But

$$
S+S f S+S=\Pi_{\alpha}\left(S_{\alpha}+S_{\alpha} f(\alpha) S_{\alpha}+S_{\alpha}\right)=\Pi_{\alpha} S_{\alpha}
$$

since for each $\alpha, S_{\alpha}+S_{\alpha} f(\alpha) S_{\alpha}+S_{\alpha}$ is a double ideal of $S_{\alpha}$ and hence equals $S_{\alpha}$. Therefore $J=\Pi_{\alpha} S_{\alpha}$.

Another method of generating doubly simple semirings is the adjunction of a 0 to a semiring. That is, if $S$ is a semiring, and an element 0 is adjoined to $S$ so that $0+x=x+0=x$ and $0 x=x 0=0$ for all $x$ in $S$, then $S \cup\{0\}$ is a doubly simple semiring. Note that if $S$ already possessed a zero, the newly adjoined one 
must be an isolated point. However, in general, a zero need not be isolated, e.g. example A(iii). Indeed, in the infinite product of semirings with zero, the product zero cannot be isolated.

If $f: R \rightarrow S$ is a continuous semiring homorphism (i.e., a continuous function between semirings which preserves both operations), and $R$ is doubly simple, then so is $f(R)$.

ExAmple B. We describe a generalization of the semigroup ring. Let $S$ be a semigroup. Let $T$ be a subset of $S$ with $T \subseteq T^{2}$ and with multiplication on $T$ finite-to-one (i.e., for each $t$ in $T,\{(a, b) \in T \times T: a b=t\}$ is a finite set). For each $x \in T$, let $R_{x}$ be a topological semiring which is additively commutative. For $a$ and $b$ in $T$ let $f_{a, b}: R_{a} \rightarrow R_{b}$ be a continuous semiring homomorphism. Suppose further that for each $a, b$, and $c$ in $T$,

$$
f_{b, c} \circ f_{a, b}=f_{a, c}
$$

For $f$ and $g$ in $\Pi_{x \in T} R_{x}$, define

$$
(f+g)(x)=f(x)+g(x)
$$

and

$$
\left(f^{*} g\right)(x)=\sum_{a b=x} f_{a, x}[f(a)] f_{b, x}[g(b)] .
$$

With these definitions, $\left(\Pi_{x \in T} R_{x},{ }^{*},+\right)$ is a topological semiring, and it is easy to check that if each $R_{x}$ has a zero (in the sense of Theorem 1) and if $f_{a, b}$ preserves zero for each $a, b$ in $T$, then $\left(\Pi_{x \in T} R_{x},{ }^{*},+\right)$ also has a zero in this sense, and hence is doubly simple.

The above situation arises in several interesting ways. Suppose $S$ and $T$ are both the additive non-negative integers. Let $R$ be a semiring with an automorphim $f$. For each $x \in S$, let $R_{x}=R$ and for each $m$ and $n$ in $S$, let $f_{n, m}=f^{m-n}$. A simpler example can be generated by taking $S=T$ to be a semigroup with $S=S^{2}$ and finite-to-one multiplication. For each $x$ in $S$ let $R_{x}=R$ be a semiring and $f_{a, b}$ the identity for each $a, b$ in $S .\left(\Pi_{x \in S} R, *,+\right)$ might be called a semigroup semiring.

Finally, let $R$ be a semiring. Consider a non-empty set $A$ and a point 0 not in $A$. Let $S=A \cup\{0\}$ with multiplication defined by $a^{2}=a$ for all $a$ in $S$ and $a b=0$ if $a \neq b$. Then $S$ is a semigroup and $A$ has the properties of $T$ above. For each $a, b$ in $A$, let $f_{a, b}$ be the identity. Then

$$
\left(\prod_{a \in A} R, *,+\right) \approx\left(\prod_{a \in A} R, \cdot,+\right)
$$

where indicates coordinatewise multiplication.

EXAMPLE C. Let $R, S$ be topological semirings and $f, g: R \rightarrow \mathrm{S}$ be two continuous semiring homorphisms. On $R \times S$, define

$$
(x, y)+(a, b)=(x+a, y+b)
$$


and

$$
(x, y)(a, b)=(x a, y f(a)+g(x) b) .
$$

Then $R \times S$ with these operations is a topological semiring and if $f$ and $g$ are zero preserving, then $R \times S$ has a zero in the sense of Theorem 1 and is doubly simple.

EXAmple D. Let $(L, \wedge)$ be a compact semilattice with isolated identity $a$. On $L \times[0,1]$, define

$$
(p, x)+(q, y)=(p \wedge q, x \wedge y) .
$$

$L \times\{0\}$ is an ideal of $L \times[0,1]$. Let $\mathscr{L}=L \times[0,1] / L \times\{0\}$, and on $\mathscr{L}$ define

$$
(a, x)(a, y)=(a, x \vee y) \text {; }
$$

let $(a, x)(p, y)=(a, x)$ if $p \neq a$; and let $(p, x)(q, y)=(p, x)+(q, y)$ if $p \neq a \neq q$. Note that $(a, 1)(x, y)=(a, 1)$ and $(a, 1)+(x, y)=(x, y)$ for all $(x, y)$ in $\mathscr{L}$. Hence $\mathscr{L}$ is doubly simple. If $L$ is $\{1,2,3$,$\} and x \wedge y$ represents $\min \{x, t\}$ then $\mathscr{L}$ is topologically a triad.

THEOREM 2. In any compact semiring $S$ there is a minimal double ideal $K$ which is compact and doubly simple.

Proof. As in the corresponding theorem for compact semigroups, $S$ has a minimal closed double ideal $K$. Now suppose $D$ is a double ideal of $S$ contained in $K$. Let $d$ be any element of $D$. Then

$$
S+S d S+S \subseteq D
$$

and $S+S d S+S$ is a closed double ideal of $S$. Thus $K=S+S d S+S \subseteq D$, so $K=D$. Therefore $K$ is a minimal double ideal.

Let $J$ be a double ideal of $K$. Note that $K+K J K+K$ is a double ideal of $S$ and is contained in $K$. Thus

$$
K=K+K J K+K \subseteq J .
$$

Therefore $K=J$ and so $K$ is doubly simple.

THEOREM 3. If $S$ is a compact semiring containing a proper double ideal $I$, then $S$ has a maximal proper double ideal $M$, and $M$ is open.

Proof. We omit the argument, which is analogous to the corresponding one for compact semigroups, but remark that the ideal generated by any set $A \subseteq S$ is $A \cup A S \cup S A \cup S A S \cup A+S \cup S+A \cup S+A+S \cup A S+S \cup S A+S \cup$ $S+A S \cup S+S A \cup S+A S+S \cup S+S A+S \cup S+S A S \cup S A S+S \cup S+$ $S A S+S$. 
REMARK. If $S$ is a connected semiring and contains a minimal double ideal ideal $K$, then $K$ is also connected.

Proof. Let $k$ be an element of $K$, and note that $S+S k S+S$ is a double ideal of $S$ contained in $K$. Thus $K=S+S K S+S$, which is the continuous image of a connected set, and hence connected.

Now suppose $S$ is a compact semiring and $J$ is a closed double ideal of $S$. Just as in the case of semigroups $[5 ; \mathrm{p}$.$] we acquire an equivalence relation \sim$ by defining $x \sim y$ if and only if $x=y$ or $x$ and $y$ are both in $J$. This equivalence is a congruence with respect to both addition and multiplication. We denote the quotient semiring $(\bmod \sim)$ by $S / J$ and notice that $J$ is a zero for the addition as well as the multiplication of $S / J$.

Thus we find that every compact semiring is associated with a doubly simple semiring $K$ and a semiring $S / K$ having a double zero. A semiring having such a double zero is easily obtained by considering the real interval $[1, \infty)$ under ordinary addition and multiplication. Compactify this semiring by adding a point $\infty$ and defining $x \infty=\infty x=x+\infty=\infty+x=\infty$ for all $x$.

Let $S$ be a compact semiring with commutative addition. Define a relation $\rho$ on $S$ by $x \rho y$ if and only if there exist $e$ and $f$ in $E[+](S)$ such that $x+e=y+f$. In [3] Bourne defines a closed congruence $\sigma$ on a semiring $S$ with commutative addition and a zero in the sense of theorem 1, by $x \sigma y$ if and only if there exists an $a$ in $S$ such that $x+a=y+a$. He shows that $S / \sigma$ is a ring. Clearly, this result does not depend on $S$ having a zero.

THEOREM 4. If $\rho$ and $\sigma$ are defined as in the preceding paragraph, then $\rho=\sigma$, for any compact additively commutative semiring.

Proof. It is easy to see that if $x \rho y$, then there exists $e$ in $E[+](S)$ such that $x+e=y+e$, which implies that $\rho \subseteq \sigma$. Now suppose $x+a=y+a$ for some $a$ in $S$. Then $x+a+a=y+a+a$, and inductively $x+n a=y+n a$ for all $n$. Let $e=e$ in $\Gamma(a)$; there is a net $\left\{n_{\alpha} a\right\}$ such that $n_{\alpha} a \rightarrow e$. Hence $x+e=\lim _{\alpha}\left(x+n_{\alpha} a\right)=\lim _{\alpha}\left(y+n_{\alpha} a\right)=y+e$. Therefore $\sigma \subseteq \rho$.

Notice that this congruence identifies the additive idempotents of $S$, suggesting that the study of compact additively commutative semirings largely reduces to the study of rings and of additively idempotent semirings. Now the additively idempotent semiring $([0,1], \cdot, \vee)$ has not only a zero in the sense of Theorem 1 but also an element 1 which is an additive zero and a multiplicative identity. Indeed we have the following.

THEOREM 5. If $S$ is a compact additively and multiplicatively commutative, additively idempotent semiring with $S^{2}=S$, then $S$ contains an element $k$ such that $k+x=k$ for all $x$ in $S$, and a unique maximal subsemiring $S^{\prime}$ such that 
$k x=x$ for all $x$ in $S^{\prime} . S^{\prime}$ is a closed subsemiring of the minimal double ideal and is connected if $S$ is connected.

Proof. $K[+]$ is $a$ group and hence contains a single point $k$. Since $S^{2}=S$, we have $S=S E[\cdot][6]$. Thus there is a multiplicative idempotent $e$ in $S$ and an element $s$ of $S$ such that $k=s e$. Now

$$
k^{2}=k(k+e)=k^{2}+k e=k^{2}+k=k,
$$

so $k$ is a multiplicative identity for $k S$, and $k S$ is a subsemiring. Let $K$ represent the minimal double ideal. Since $\{k\}$ and $K$ are additive ideals, $\{k\}$ meets $K$; that is $k \in K$. But $K$ is a multiplicative ideal, so $k S \subseteq K$. Suppose $C$ is a subsemiring containing $k$ as a multiplicative identity. For each $y$ in $C$ we have $y=k y \in k S$, i.e., $C \subseteq k S$. Thus $k S$ is the unique maximal such semiring. Clearly $k S$ is closed and is connected if $S$ is connected.

Even if $S$ has a multiplicative identity 1 , it need not be $k$. This can be seen in the semigroup semiring of $([0,1], \cdot V)$ over $Z_{2}$ (the integers mod 2), that is $[0,1]$ $\times[0,1]$ with

$$
(x, y)(a, b)=(x a \vee y b, y a \vee x b) .
$$

Here $k=(1,1), 1=(1,0)$ and $S^{\prime}$ is the diagonal.

It would be interesting to know the structure of the $S^{\prime}$ of the last theorem.

\section{References}

[1] S. Bourne, 'The Jacobson radical of a semiring,' Proc. Nat. Acad. Sci. U.S.A. 37 (1951), $163-170$.

[2] S. Bourne, 'On multiplicative idempotents of a potent semiring,' Proc. Nat. Acad. Sci. U.S.A. 42 (1956), 632-638.

[3] S. Bourne, 'On compact semirings,' Proc. Jap. Acad., 35 (1959), 332-334.

[4] A. D. Wallace, 'The structure of topological semigroups,' Bull. Amer. Math. Soc. 61 (1955), 95-112.

[5] K. H. Hofmann and P. S. Mostert, Elements of Compact Semigroups (Columbus, Ohio, 1966).

[6] A. B. Paalman DeMiranda, Topological Semigroups (Mathematisch Centrum, Amsterdam 1964), 45.

Department of Mathematics

Clarkson College of Technology

Potsdam, New York 